Edith Cowan University

Research Online

ECU Publications Post 2013

$10-1-2018$

\title{
Statistical analysis plan (SAP) for the Very Early Rehabilitation in Speech (VERSE) after stroke trial: an international 3-arm clinical trial to determine the effectiveness of early, intensive, prescribed, direct aphasia therapy
}

\author{
Erin Godecke \\ Edith Cowan University \\ Tapan Rai \\ Dominique A Cadilhac \\ Elizabeth Armstrong \\ Sandy Middleton
}

See next page for additional authors

Follow this and additional works at: https://ro.ecu.edu.au/ecuworkspost2013

Part of the Neurology Commons

\section{$10.1177 / 1747493018790055$}

This is an Author's Accepted Manuscript of: Godecke, E., Rai, T., Cadilhac, D. A., Armstrong, E., Middleton, S., Ciccone, N., ... \& Hankey, G. J. (2018). Statistical analysis plan (SAP) for the Very Early Rehabilitation in Speech (VERSE) after stroke trial: an international 3-arm clinical trial to determine the effectiveness of early, intensive, prescribed, direct aphasia therapy. International Journal of Stroke, 13(8), 863-880. Available here.

This Journal Article is posted at Research Online.

https://ro.ecu.edu.au/ecuworkspost2013/5173 


\section{Authors}

Erin Godecke, Tapan Rai, Dominique A Cadilhac, Elizabeth Armstrong, Sandy Middleton, Natalie Ciccone, Anne Whitworth, Miranda L Rose, Audrey Holland, Fiona Ellery, Graeme J Hankey, and Julie Bernhardt 


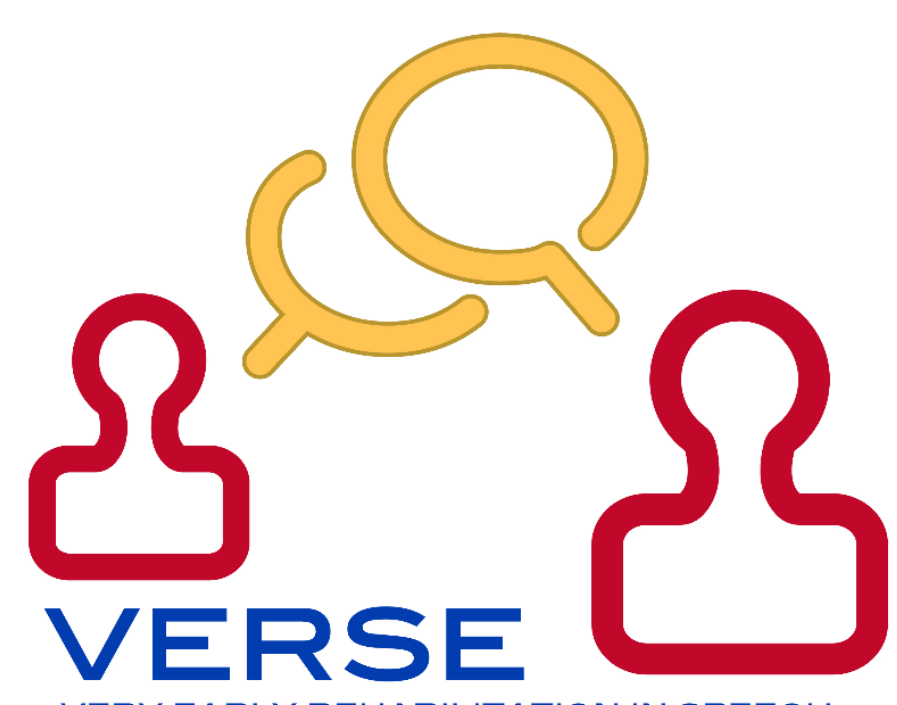

VERY EARLY REHABILITATION IN SPEECH

\title{
Statistical Analysis Plan
}

\section{March 2018}

Erin Godecke, Tapan Rai, Dominique A Cadilhac, Elizabeth Armstrong, Sandy Middleton, Natalie A. Ciccone, Anne Whitworth, Miranda L. Rose, Audrey Holland, Fiona Ellery, Graeme J Hankey, Julie Bernhardt for the VERSE Collaboration.

\author{
Author for correspondence: \\ A/Professor Erin Godecke \\ School of Medical and Health Science \\ Edith Cowan University \\ 270 Joondalup Dve \\ JOONDALUP WA 6027 T +61 863045901 \\ e.godecke@ecu.edu.au
}




\section{Table of Contents}

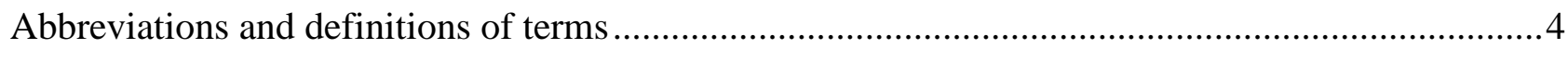

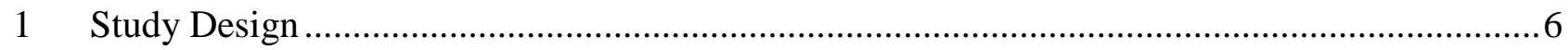

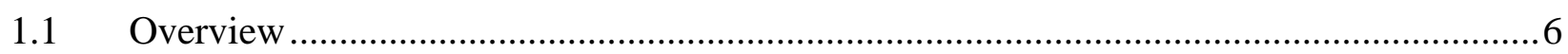

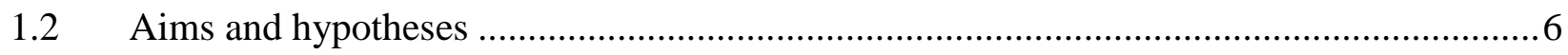

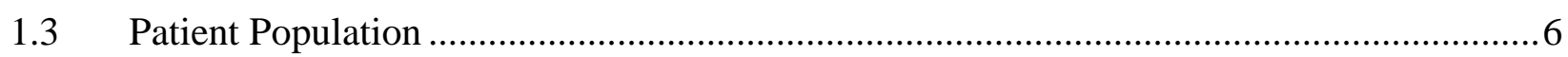

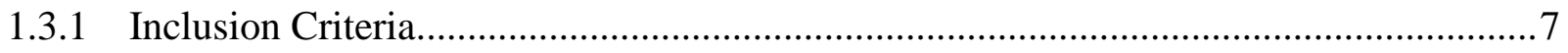

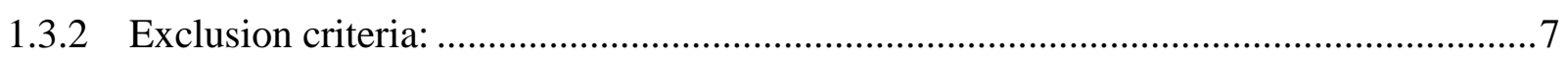

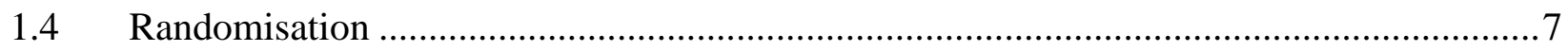

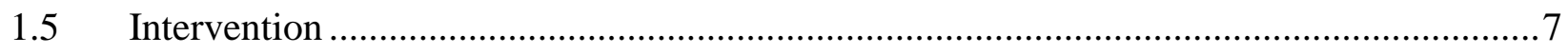

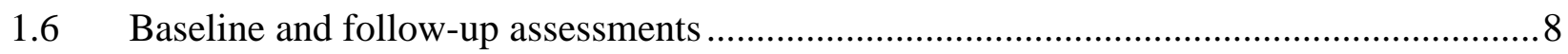

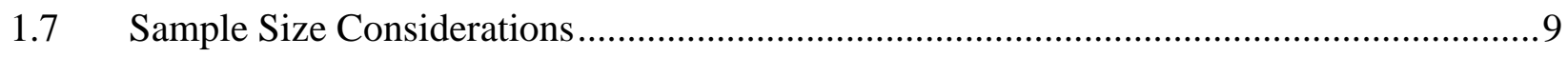

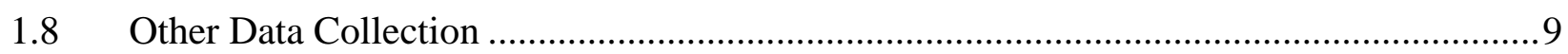

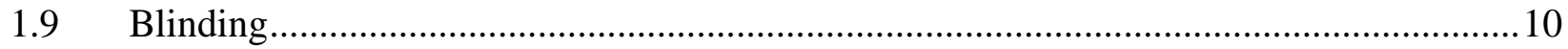

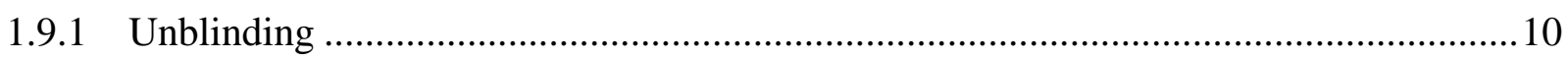

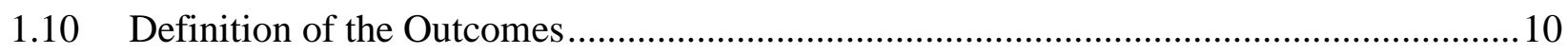

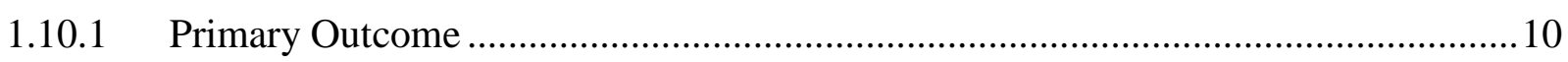

1.10.2 Secondary Outcome Measures ...................................................................... 10

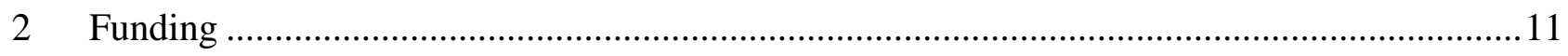

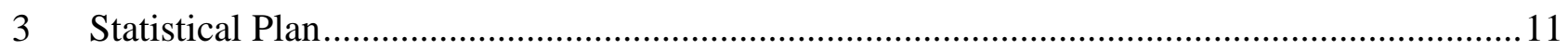

3.1 Analysis principles and general considerations ..........................................................11

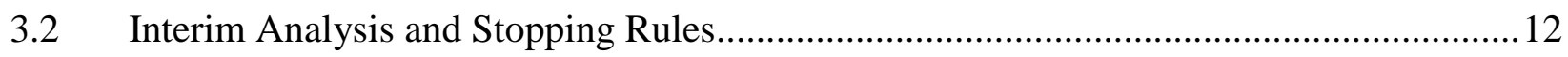

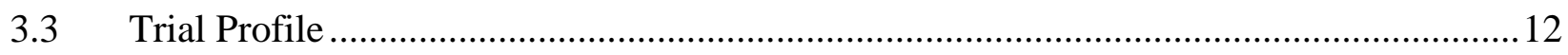

3.4 Patient Characteristic and baseline comparisons ......................................................... 12

3.5 Primary Outcome: Communication Recovery at 12 weeks by Intensity ..........................12

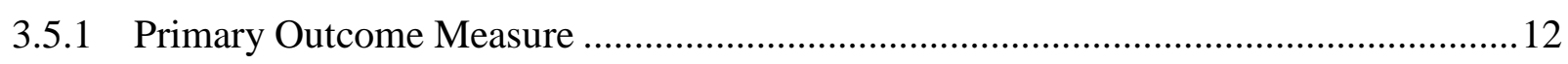

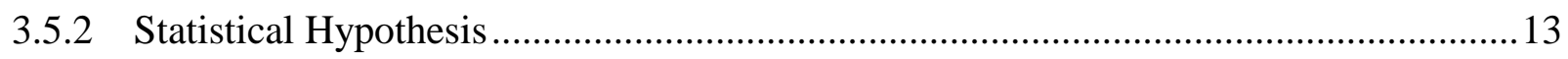

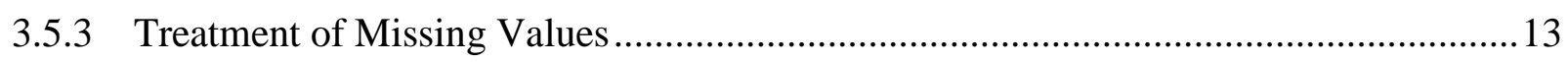

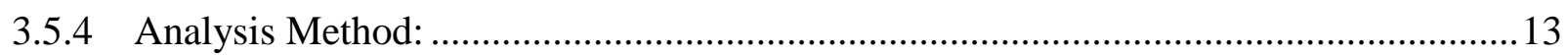

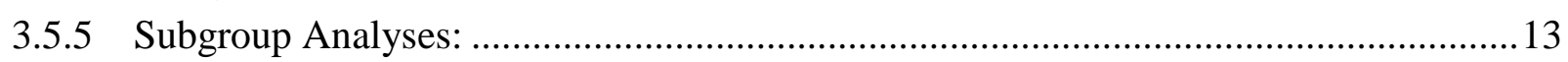

3.6 Secondary Effectiveness Hypotheses: ..................................................................... 14

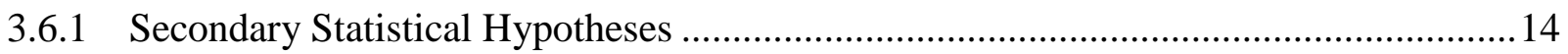

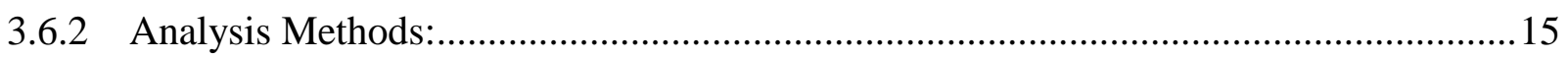

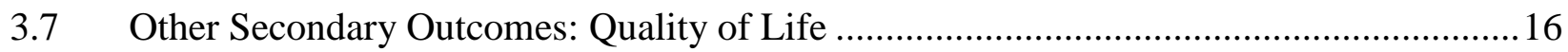

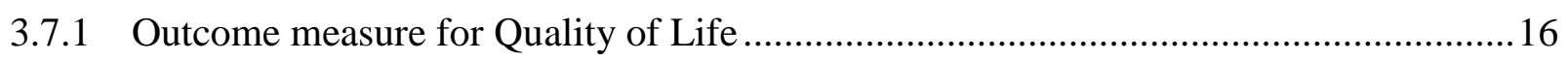

3.7.2 Statistical Hypotheses for Quality of Life................................................................. 16

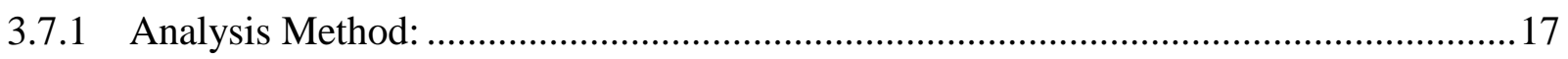

3.8 Other Secondary Outcomes: Cost Effectiveness ….......................................................17

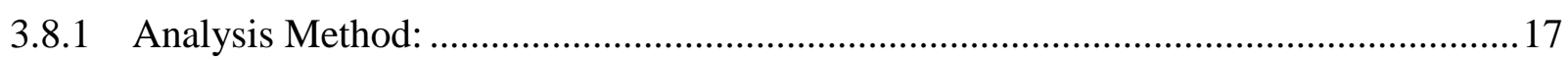

3.9 Secondary Outcomes: Safety Important Medical Events (IME) Adverse Events (AE) and

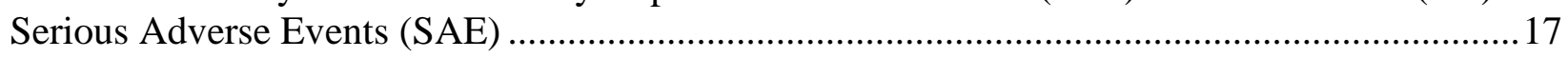

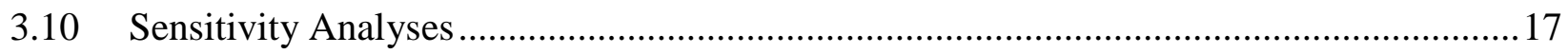

3.11 Tables and Figures for the Main Paper ................................................................... 18 


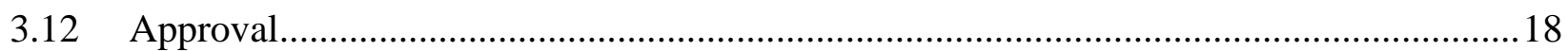

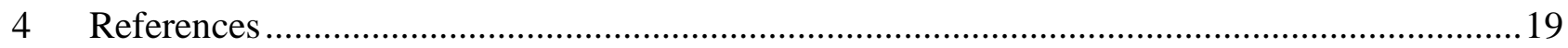

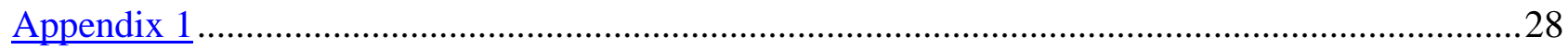




\section{Abbreviations and definitions of terms}

\begin{tabular}{|c|c|}
\hline ADRS & Aphasia Depression Rating Score \\
\hline $\mathrm{AE}$ & Adverse Event \\
\hline ACTRN & Australasian Clinical Trial Registry Number \\
\hline $\mathrm{AQ}$ & Aphasia Quotient (derived from Western Aphasia Battery) \\
\hline AusTOMS & Australian Therapy Outcome Measures \\
\hline BNT & Boston Naming Test \\
\hline CIUs & Correct Information Units \\
\hline CONSORT & Consolidated Standards of Reporting Trials \\
\hline $\mathrm{CRF}$ & Case Report Form \\
\hline CTA & Clinical Trial Agreement \\
\hline DSMC & Data Safety Monitoring Committee \\
\hline eCRF & Electronic Case Report Form \\
\hline ECU & Edith Cowan University \\
\hline FAST & Frenchay Aphasia Screening Test \\
\hline FDA & Food and Drug Administration \\
\hline GCP & Good Clinical Practice \\
\hline GEE & Generalised Estimating Equations \\
\hline GLMM & Generalised Linear Mixed Model \\
\hline GP & General Practitioner \\
\hline IEC & Independent Ethics Committee \\
\hline IME & Important Medical Event \\
\hline IQR & Interquartile Range \\
\hline LMM & Linear Mixed Model \\
\hline mRS & Modified Rankin Score \\
\hline MAR & Missing at Random \\
\hline NHMRC & National Health and Medical Research Council \\
\hline NIHSS & National Institute of Health Stroke Score \\
\hline NTA & Neuroscience Trials Australia \\
\hline OCSP & Oxfordshire Community Stroke Project Classification \\
\hline RBHOM & Royal Brisbane Hospital Outcome Measure for Swallowing \\
\hline RCT & Randomised Controlled Trial \\
\hline SAE & Serious Adverse Event \\
\hline SAQoL-39 & Stroke and Aphasia Quality of Life \\
\hline SAH & Sub Arachnoid Haemorrhage \\
\hline SD & Standard Deviation \\
\hline SDH & Sub Dural Haemorrhage \\
\hline TIA & Transient Ischemic Attack \\
\hline TGA & Therapeutic Goods Administration \\
\hline UC & Usual Care \\
\hline UTN & Unique Trial Number \\
\hline VERSE & Very Early Rehabilitation in SpEech \\
\hline
\end{tabular}


WAB- $\mathrm{R}$

$\% \mathrm{MPR}$
Western Aphasia Battery-Revised

Percent of Maximal Potential Recovery 


\section{Study Design}

\subsection{Overview}

VERSE $^{1}$ is a prospective, parallel group, assessor blinded, randomised, multicentre, international clinical trial. Ethics and local governance approval was obtained from all sites participating. Version 3 of the protocol is current at the time of publication. Written, informed consent is obtained from each participant or their legal representative using full hospital approved consent processes supported by aphasia friendly consent documents. All participants in VERSE receive usual ward based care. Participants are randomised in a ratio of 1:1:1 to one of three groups (i) usual ward care alone (UC); or (ii) usual ward care plus high intensity aphasia therapy (UC-Plus); or (iii) usual ward care plus high intensity prescribed and structured aphasia therapy (VERSE). The distinguishing features of each of these arms is described in Section 1.5.

\subsection{Aims and hypotheses}

The primary aim of the VERSE trial is to determine whether very early daily aphasia therapy (UCPlus and VERSE groups) is superior to usual ward care at 12 weeks post stroke. The secondary aim is to test whether a standardised intense aphasia therapy regimen (VERSE) is more effective and cost effective than non-standardised intense aphasia therapy (UC-Plus). Participants are recruited within the first 14 days following stroke. For the purposes of this trial, aphasia therapy refers to direct aphasia treatment designed to change the communication performance of the person with aphasia. All analyses herewith include only direct aphasia therapy which does not include patient/family counselling and education, case management or documentation.

\section{Primary Hypothesis:}

Very early aphasia therapy will result in greater improvement in communication at 12 weeks poststroke, as measured by the Western Aphasia Battery-Revised; Aphasia Quotient (WAB-R (AQ)). ${ }^{2}$

\section{Secondary Hypotheses:}

1. VERSE aphasia therapy will result in a greater improvement in communication than UC-Plus aphasia therapy at 12 and 26 weeks post stroke, as measured by the WAB-R (AQ). ${ }^{2}$

2. VERSE aphasia therapy and UC-Plus will each result in greater improvements in communication than UC alone, as measured by the WAB-R(AQ) ${ }^{2}$, at 12 weeks and 26 weeks post stroke.

3. VERSE aphasia therapy will result in greater improvements in connected speech than UCPlus, as measured by Discourse Analysis, at 12 weeks and 26 weeks post stroke.

4. VERSE aphasia therapy and UC-Plus will each result in greater improvements in connected speech than UC alone, as measured by Discourse Analysis, at 12 weeks and 26 weeks post stroke.

5. Very early aphasia therapy (UC-Plus and VERSE) will result in better quality of life at 12 and 26 weeks post stroke than UC control.

6. Very early aphasia therapy (UC-Plus and VERSE) will be more cost-effective than UC at 12 and 26 weeks post stroke.

\subsection{Patient Population}

Participants include patients with acute stroke and aphasia screened using the shortened Frenchay Aphasia Screening Test. ${ }^{3}$ Participants are identified, recruited, randomised and assessed within 14 days of stroke. Therapy commences the day after this assessment. 


\subsubsection{Inclusion Criteria}

Patients are eligible if the following inclusion criteria are met:

- The patient has acute aphasia of any type resulting from an acute stroke (ICD 10 codes 161 164 i.e. no TIA, SAH or SDH);

- The patient has a score of less than 93.7 of the Aphasia Quotient of the Western Aphasia Battery-Revised ${ }^{2}$

- The patient is deemed medically stable at recruitment

- The patient can maintain a wakeful alert state for 30 consecutive minutes within 14 days of stroke onset

- The patient has normal or corrected hearing and vision

- The patient is at least 18 years old; there is no upper age limit for inclusion.

- Informed consent is obtained from the patient or person responsible

\subsubsection{Exclusion criteria}

Patients are excluded from the study if they have any of the following:

- Pre-existing aphasia before the stroke event

- Have suffered a head injury or have had or require neurosurgery (clot retrieval surgery is not an exclusion if patient is stable post operatively and meets the above criteria)

- Pre-existing clinical diagnosis of dementia

- Diagnosis or treatment of major depression at the time of enrolment

- Concurrent progressive neurological disorders

- Unable to participate in English based therapy due to English being a second language.

- Is participating in another intervention trial, which in the opinion of the VERSE trial manager, may interfere with the outcome of the VERSE trial.

\subsection{Randomisation}

Participants are randomised to one of three groups (UC, UC-Plus, VERSE) via a central allocation system in a 1:1:1 ratio. Randomisation is stratified by aphasia severity on the WAB-AQ ${ }^{2}$ (Severe: WAB-AQ2 0-31.2; Moderate: WAB-AQ2 31.3-62.5; Mild: WAB-AQ² 62.6-93.7). A remote, webbased, computer-generated randomisation procedure is used. All online data submissions are secured by use of password site entry and data encryption procedures. Once the site staff submits patient recruitment data, the randomisation allocation is immediately provided back to the investigator.

\subsection{Intervention}

Participants randomised to one of three arms of therapy receive direct aphasia therapy with a qualified speech pathologist for a maximum period of 25 working days. The three arms are:

1 Usual Care: Usual ward based aphasia care provided as per health care site standards.

2 UC-Plus: Usual ward based care for aphasia is provided but using a defined therapy regimen of daily sessions of 45 - 60 minutes; each participant receives 20 sessions (15-20 hours of direct aphasia therapy) in addition to usual care.

3 VERSE intervention: The intensity of this arm of therapy will match the Usual Care-Plus arm, (20 daily sessions of 45 - 60 minutes duration; 15 - 20 hours of direct aphasia therapy) but the speech and language training is prescribed and standardised according to a separate VERSE intervention protocol, in addition to usual care. 
The intervention period for the trial concludes at a maximum of 50 days post stroke or when 20 sessions of aphasia therapy are completed (UC-Plus and VERSE) - whichever comes first. Participants are followed up at 12 weeks and 26 weeks post stroke.

Details of the intervention are provided in the VERSE Trial protocol (IJS, 2016).

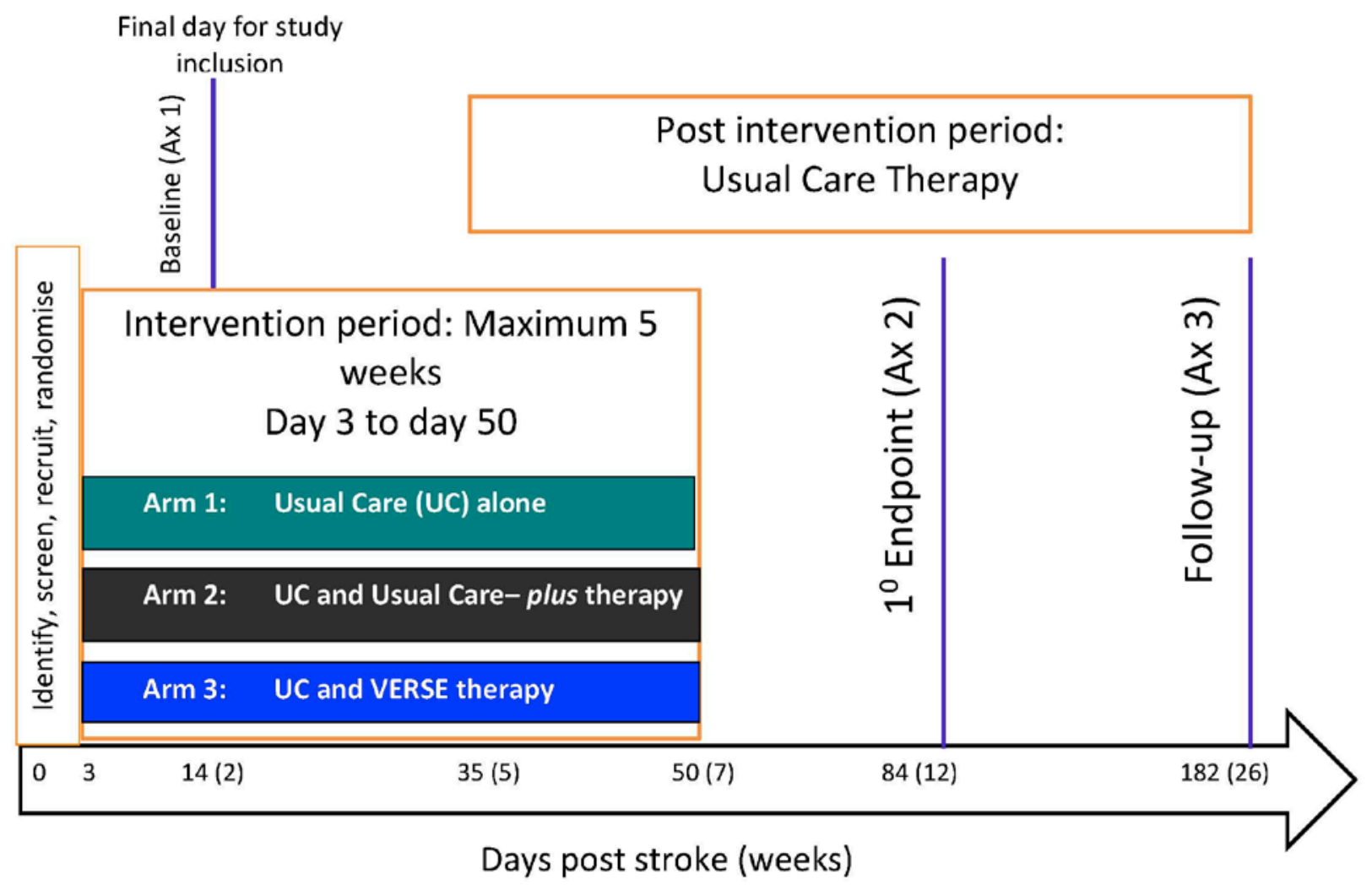

Figure 1. Trial timeline

\subsection{Baseline and follow-up assessments}

All stroke patients with aphasia, who are at least 18 years old, are screened for inclusion in the study. Those screened and deemed ineligible for the study are entered onto a screening log which outlines the reason for non-inclusion. Details of the screening and recruitment procedures, as well as the assessment schedule are included in the main trial protocol.

The assessment timeline is outlined in Figure 1. Baseline assessment occurs no earlier than two days from the onset of stroke and no later than 14 days from the onset of stroke. Participants undergo the (AQ) component of the WAB-R. ${ }^{2}$ Participants are also assessed for their medical and stroke history. Baseline data collection includes the collection and documentation of:

1) Demographic details including age, gender, past medical history, languages spoken, education level, handedness, employment, Aboriginal or Torres Strait islander status and living arrangements.

2) Stroke type and severity (type, hemisphere, first or recurrent stroke, National Institute of Health Stroke Scale, (NIHSS ${ }^{4}$ ), modified Rankin Scale $\left(\mathrm{mRS}^{5}\right.$ ), Oxfordshire Community Stroke Project Classification $\left(\mathrm{OCSP}^{6}\right)$, Royal Brisbane Hospital Outcome Measure for Swallowing $\left(\mathrm{RBHOM}^{7}\right)$ and Australian Therapy outcome measures (AusTOMS ${ }^{8}$ ).

3) Additional baseline assessments including the Boston Naming Test $\left(\mathrm{BNT}^{9}\right)$, the "clock drawing" cognitive sub test, and discourse collection.

4) Time of assessment and aphasia therapy commencement. 
Therapy commences the day after the baseline assessment, and is completed within a maximum of 25 working days and must be completed by day 50 post stroke.

The participants are followed up at their place of residence or in the hospital clinic by a blinded assessor, 12 weeks and 26 weeks after the date of the stroke. Patient assessments at these follow-up visits include:

- the Aphasia Quotient component of the Western Aphasia Battery-Revised ${ }^{2}$

- $\quad$ the Boston Naming Test, ${ }^{9}$

- Discourse collection, ${ }^{10-16}$

- Stroke and Aphasia Quality of life questionnaire 39 item version ${ }^{17}$; and

- $\quad$ The Aphasia Depression Rating Scale. ${ }^{18}$

\subsection{Sample Size Considerations}

The study is powered to detect a difference of $4.4 \%$ in communication improvement, measured as percent of maximal improvement (\%MPR) on the WAB-AQ. ${ }^{2}$ This is based on the smallest difference between VERSE and UC-plus estimated from our pilot studies. We have consulted international aphasia experts who have reported a previous consensus that a 5-point difference on the WAB-AQ ${ }^{2}$ is clinically meaningful improvement in communication. It is important to note this relationship is not linear and will vary based on aphasia severity. We believe the $4.4 \%$ change in communication status, which is marginally more conservative than the 5-point difference reported in consensus is reasonable, given this is the difference between the two intensive arms of this trial. The required sample size was estimated under the assumption that the effectiveness hypotheses would be analysed using a longitudinal regression model (generalised estimating equations or linear mixed model), with outcomes measured at three times (at baseline, 12 and 26 weeks months).

A sample of 246 participants (82 per arm) will provide $80 \%$ power to detect this difference at a (twosided) significance level of $\alpha=0.05$, after adjusting for a 20\% loss to analysis of (lost to follow-up due to death, dropout and non-adherence).

Our pilot data shows similar effect sizes for differences between the VERSE and UC-plus interventions for discourse measures and communication outcomes. Therefore, we believe that the sample size will provide sufficient power to assess differences in discourse outcomes. Pilot data on quality of life for this population was not available at the trial inception however, we expect to observe larger effects on quality of life than on communication or discourse and therefore expect to have sufficient power to assess difference on quality of life.

\subsection{Other Data Collection}

During the intervention period, all Usual Care speech therapy is documented in medical notes. The detail of all additional trial related aphasia therapy is documented separately on the electronic Case Report Form (eCRF) as part of the clinical trial. A daily speech therapy log is completed for each trial participant which includes content, duration of session and frequency of sessions. Where usual care sessions with participants have occurred prior to the participant's enrolment in the trial, these sessions are retrospectively collected from the participant's medical record to ensure all therapy provided to the participant enrolment is captured. Deviation from the prescribed therapy intensity protocol (UC-Plus and VERSE), is documented. Reasons for withdrawal of therapy such as health related complications, patient withdrawal and death are recorded. At the end of the treatment period, a participant diary is provided to the patient to capture resource utilisation information.

Participants are assessed and questioned about general health to determine if there have been any adverse or Serious Adverse Events during the study period.

The assessment visits (Baseline, Week 12 and Week 26) are completed as a single session. If the participant is unable to complete assessment tasks in a single session, they are completed on the same 
day if possible (ie: over a morning and afternoon session). If this is not possible, the assessments take place on consecutive days and a protocol deviation is recorded.

Information relating to resource utilisation is obtained by the blinded assessor from the participant and the participant diary and includes general practitioner (GP) visits, hospital and rehabilitation admissions, outpatient and community service use, medication use for anxiety and depression, speech therapy, use of speech aids and devices, employment status, respite care and informal care as a result of stroke. Where possible, resource utilisation data is verified in medical or other hospital records.

\subsection{Blinding}

VERSE is a complex intervention and therefore, it is difficult to blind the participants to receipt of intense treatment or usual care. Similarly, it is not possible to blind the clinician providing therapy to the participant's treatment allocation. Every effort is made to ensure only minimal staff are aware of randomisation allocation, and all outcome assessments are conducted by assessors who are blinded to allocation. In addition, chief investigators and trial statistician are blinded to treatment allocation.

\subsubsection{Unblinding}

Only the Data and Safety Monitoring Committee (DSMC) have access to progressive data. The DSMC Chair is Professor Richard Lindley, University of Sydney, NSW, Australia. The DSMC review unblinded data in accordance with the DSMC Charter (Version 1.0, 25 February 2015).

\subsection{Definition of the Outcomes}

\subsubsection{Primary Outcome}

The primary outcome is improvement in communication at 12 weeks after stroke. Communication outcome is measured by the Western Aphasia Battery- Revised Aphasia Quotient (WAB-R (AQ)). ${ }^{2}$ Improvement will be assessed using the percent of maximal potential recovery achieved (\%MPR). ${ }^{19}$ A formal definition of \%MPR ${ }^{19}$ is provided in Section 3.5.1.

\subsubsection{Secondary Outcome Measures}

The secondary outcome measures include effectiveness, quality of life, safety and cost effectiveness measures. These are:

- Communication improvement at 26 weeks, assessed as $\% \mathrm{MPR}^{19}$ at 26 weeks

- Improvement in connected speech at 12 and 26 weeks on discourse measures ${ }^{10-16}$

- Quality of life measured by the Stroke and Aphasia Quality of Life (SAQOL) ${ }^{17}$ scale

- Depression measured at 12 and 26 weeks by the Aphasia Depression Rating Scale (ADRS) ${ }^{18}$

- Adverse Events and serious adverse events as defined in the trial protocol

- Cost effectiveness of VERSE and UC-Plus therapies compared to Usual Ward Care. The economic analysis will be outlined separately in an Economic Analysis Plan 


\section{Funding}

This study is funded by the National Health and Medical Research Council (NHMRC), Australia (Project Grant Number 1044973), The Tavistock Trust for Aphasia, United Kingdom and Edith Cowan University, Australia. The funders of this study have no influence on the study design, data collection, data interpretation, writing of the protocol or statistical analysis plan.

\section{Statistical Plan}

\subsection{Analysis principles and general considerations}

- All outcomes and analyses are prospectively characterised as primary or secondary.

- Differences in all endpoints between the three arms of the trial (VERSE, UC-Plus, UC) will be tested independently at the two-tailed 5\% significance level. All estimates of treatment effects will be presented with 95\% confidence intervals.

- No formal adjustments will be undertaken to constrain the Type I error associated with planned secondary or exploratory analyses. The information provided by analyses is designed to supplement the evidence from the primary analyses; it will provide a more complete characterization of the treatment effects.

- The analyses for all outcome measures (effectiveness, quality of life and safety) will be conducted on an intention the treat (ITT) basis i.e. all patients will be analysed as members of the group to which they were randomised, irrespective of whether they received the allocated treatment or not. The ITT strategy for VERSE is based on the following principles:

o All available outcome data are collected on all randomised patients.

o All participants are analysed in the groups to which they are randomised.

o All available outcome data will be used in the primary analyses. The primary analyses will be reported without missing data imputed.

o A separate analysis with missing data imputed will also be reported. Missing data will be assumed to be missing at random (see section 3.5.3)

o A sensitivity analysis including all randomised individuals will be conducted. The sensitivity analysis will consider alternative assumptions about data missing not at random (MNAR) (see section 3.10).

- A per-protocol analysis will be conducted separately, but will not be included in the primary results for this trial. The per-protocol analysis will be described in greater detail when it is published. However, it will be based on the following principles:

o The per-protocol cohorts will be based on whether or not participants received the planned minimum 15 hours of direct aphasia therapy as the intervention dose.

o The per-protocol cohort will include:

- VERSE participants who receive at least 15 hours of direct language therapy over the intervention period;

- UC-Plus participants who receive at least 15 hours of direct language therapy over the intervention period;

- All UC participants who are randomised to the UC group.

- For primary and secondary analyses, the treatment effects for the primary effectiveness outcomes will be adjusted for baseline aphasia severity, measured by the WAB-R (AQ) ${ }^{2}$ and baseline stroke severity measured with the NIHSS. ${ }^{4}$ Unadjusted analyses will be reported separately from these pre-specified analyses. 
- Subgroup analyses will be carried out irrespective of whether there is a significant treatment effect on the primary outcome. Their purpose is to supplement evidence from the primary analyses to help to fully characterize the treatment effect. Results from subgroup analyses will be interpreted in this context.

- Analyses will primarily be conducted using the R Statistical Programming Language ${ }^{20}$ and supplemented with SPSS ${ }^{21}$ to generate formatted tables.

\subsection{Interim Analysis and Stopping Rules}

There are no formal interim analyses planned for this trial. The DSMC periodically reviews data for the primary outcome measure and safety, and will advise the chair of the Executive committee if, in their view, the randomised comparisons have provided both (i) 'proof beyond reasonable doubt' that very early aphasia rehabilitation (VERSE and UC-Plus groups) is clearly indicated or clearly contraindicated because of safety concerns and (ii) evidence that might influence future patient management. The DSMC are guided by Haybittle-Peto boundaries in making this determination. That is, they work on the principle that a difference of at least 3 standard errors in the analysis of effectiveness or serious adverse events (e.g. death from all causes, aspiration pneumonia within the first 50 days post stroke) may be needed to justify halting, or modifying the study before the planned recruitment is completed.

\subsection{Trial Profile}

The trial will be reported in accordance with the CONSORT statement for non-pharmaceutical trials ${ }^{19}$ and the VERSE therapeutic protocol conforms to the SPIRIT statement. ${ }^{22,23}$ The report will include the number of screened patients who met the inclusion criteria, the number included, and the major reasons for exclusion of eligible patients. At follow up, the number of patients withdrawn, lost to follow up and the number who died within that period will be reported.

\subsection{Patient Characteristic and baseline comparisons}

Baseline participant and stroke characteristics will be presented for each of the three groups: UC, UC-Plus and VERSE (Table 1). These will include: age, gender, geographic region (Australia/NZ), stroke risk factors, stroke type, stroke severity (NIHSS), ${ }^{4} \mathrm{mRS},{ }^{5}$ time to randomisation and baseline assessment. Baseline communication, cognition and swallowing characteristics will be presented in Table 2 and will include Aphasia severity, as measured by the WAB-R (AQ), ${ }^{2}$ clock drawing test, Discourse Scores (words, correct information units), ${ }^{11-16}$ AuSTOMS, ${ }^{8}$ and RHBOMS. ${ }^{7}$

Discrete variables will be summarized as frequencies and percentages. Unless otherwise indicated in the tables, percentages will be calculated according to the number of patients for whom data are available. If there are more than 5\% missing values, the denominator will be indicated in the corresponding summary table. Continuous variables will be summarized by the mean and standard deviation (SD) or by the median and interquartile range (IQR). Durations and time intervals will be summarized by medians and IQRs.

\subsection{Primary Outcome: Communication Recovery at 12 weeks by Intensity}

\subsubsection{Primary Outcome Measure}

The primary outcome is improvement in communication at 12 weeks after stroke. Communication outcome will be measured by the WAB-R (AQ). ${ }^{2}$ The WAB-R (AQ) is a comprehensive measure incorporating production of spoken language and auditory comprehension. Improvement will be assessed using the percent of maximal potential recovery achieved (\%MPR), ${ }^{19}$ which is calculated as the ratio of the difference in WAB-R (AQ) ${ }^{2}$ at 12 weeks and WAB-R $(A Q)^{2}$ at baseline, divided by the difference of maximal attainable score on WAB-R $(A Q)^{2}$ and the WAB-R $(A Q)^{2}$ at baseline. That is, 


$$
\begin{aligned}
\% M P R \text { at } 12 \text { weeks } & =\frac{(A Q \text { at } 12 \text { weeks })-(A Q \text { at baseline })}{(\text { Maximum attainable } A Q \text { score })-(A Q \text { at baseline })} \times 100 \\
& =\frac{(A Q \text { at } 12 \text { weeks })-(A Q \text { at baseline })}{100-(A Q \text { at baseline })} \mathrm{X} 100
\end{aligned}
$$

\subsubsection{Statistical Hypothesis}

The primary research hypothesis is that:

Compared to usual care alone, providing very early daily aphasia therapy (UC-Plus or VERSE) will result in greater improvements in communication ability at 12 weeks post stroke. This will be tested by refuting the null hypothesis that there is no difference in communication recovery, between the usual care group (UC) and the groups receiving very early intensive therapy (UC-Plus or VERSE) at 12 weeks post stroke. For purpose of testing this intensity hypothesis, the UC-Plus and VERSE groups will be combined into a single high intensity group.

\subsubsection{Treatment of Missing Values}

The primary analyses will be presented both with and without imputation of missing data. The imputation will treat missing values as missing at random (MAR). i.e. it will be assumed that the missing data are related to observed data, but not related to the value of the missing data itself. That is, it is assumed that the values of the missing data may reasonably be predicted from all observed data. In particular, it will be assumed that missing values of the primary outcome measure (WAB-R $(\mathrm{AQ})^{2}$ at 3 months) may be estimated from variables on which data has been collected (e.g. baseline aphasia severity, baseline stroke severity, age, gender, lesion size and location), and on the observed values of WAB-R (AQ). ${ }^{2}$ Multiple imputations will be conducted using chained equations. ${ }^{22} \mathrm{~A}$ separate model will be developed for each imputation. The pooled result of these imputed models will be reported and compared with the primary model (without imputed data).

Based on monitoring by the DSMC, $13 \%$ of the data for the primary outcome measure in the VERSE trial is missing at the time of publication of the statistical analysis plan. This is substantially less than in previous aphasia trials and less than the $20 \%$ missing data that had been planned for in the planning of this trial. ${ }^{1}$ The assumption that the data are missing at random was discussed by the VERSE Executive Committee as well as the DSMC, and considered to be plausible based on the information available at the time. Sensitivity analyses that consider various other plausible assumptions about missing data will be presented (see below section 3.10, page16).

\subsubsection{Analysis Method:}

The primary aim of this trial is to test the effect of intensity of therapy in early stroke recovery. This is achieved through the primary effectiveness hypothesis which will be analysed using a linear mixed effects regression model with $\% \mathrm{MPR}^{16}$ as the outcome measure. The UC-Plus and VERSE groups will be combined into a single high intensity group. This combined intervention group will be compared to the UC group on the primary outcome measure (\%MPR ${ }^{16}$ at 12 weeks). The model will adjust for differences in baseline aphasia severity and baseline stroke severity by including the baseline WAB-R(AQ) ${ }^{2}$ score and the baseline $\mathrm{NIHSS}^{4}$ score as covariates in the model. The effect of hospital site will be controlled for by including hospital site as a random effect. The treatment effect will be reported as difference in \%MPR with the corresponding 95\% confidence interval.

Since publication of the trial protocol ${ }^{1}$ five new sites have been added to address low participant recruitment. As a result, the Executive Committee decided there was a need to include hospital site as a random effect. This required the adaptation of our original statistical plan from using General Estimating Equations model (GEE) to a Linear Mixed Effect Regression models.

\subsubsection{Subgroup Analyses:}

The linear mixed effects regression model will be modified to analyse the difference in the primary effectiveness outcome (\%MPR ${ }^{16}$ at 12 weeks) between 
1) the VERSE group and UC group AND

2) the UC- Plus group and the UC group

As with the primary analysis, the model will adjust for differences in baseline aphasia severity and baseline stroke by including the baseline WAB-R(AQ) ${ }^{2}$ score and the baseline NIHSS ${ }^{4}$ score as covariates in the model. The effect of hospital site will be controlled for by including recruiting site as a random effect. The main between-group difference at each time point will be assessed through the interaction effect of group and timepoint. The treatment effect for each subgroup analysis will be reported as difference in $\% \mathrm{MPR}^{16}$ with the corresponding $95 \%$ confidence interval.

No corrections for multiple testing in subgroup analyses will be undertaken for these planned analyses. The unadjusted p-values will be reported together with the number of subgroup analyses undertaken.

\subsection{Secondary Effectiveness Hypotheses:}

The following secondary effectiveness hypotheses will be assessed:

1. VERSE (standardised, daily very early) aphasia therapy will result in greater improvements in communication ability than UC-Plus (non-standardised daily very early), as measured by the WAB-R (AQ), ${ }^{2}$ at 12 weeks and 26 weeks post stroke.

2. VERSE (standardised, daily very early) aphasia therapy and UC-Plus (non-standardised daily very early) will each result in greater improvements in communication ability than Usual Care alone (UC), as measured by the WAB-R (AQ), ${ }^{2}$ at 12 weeks and 26 weeks post stroke.

3. VERSE (standardised, daily very early) aphasia therapy will result in greater improvements in connected speech than UC-Plus (non-standardised daily very early) as measured on Discourse Analysis ${ }^{10,11}$ (number of words, number of Content information units; \% Content information units), at 12 weeks and 26 weeks post stroke.

4. VERSE (standardised, daily very early) aphasia therapy and UC-Plus (non-standardised daily very early) will each result in greater improvements in connected speech than Usual Care alone (UC), as measured by Discourse Analysis ${ }^{10-16}$ (number of words, number of Content information units; \% Content information units), at 12 weeks and 26 weeks post stroke.

\subsubsection{Secondary Statistical Hypotheses}

The specified set of secondary effectiveness hypotheses involve the assessment of the following statistical hypotheses:

1. VERSE (standardised, daily very early) aphasia therapy will result in greater improvements in communication ability than UC-Plus (non-standardised daily very early), as measured by the WAB-R (AQ), ${ }^{2}$ at 12 weeks post stroke.

2. VERSE (standardised, daily very early) aphasia therapy will result in greater improvements in ability than UC-Plus (non-standardised daily very early), as measured by the WAB-R (AQ), ${ }^{2} 26$ weeks post stroke.

3. VERSE (standardised, daily very early) aphasia therapy will result in greater improvements in communication ability than Usual Care alone (UC), as measured by the WAB-R (AQ), ${ }^{2} 12$ weeks post stroke.

4. VERSE (standardised, daily very early) aphasia therapy will result in greater improvements in communication ability than Usual Care alone (UC), as measured by the WAB-R (AQ), ${ }^{2} 26$ weeks post stroke.

5. UC-Plus (non-standardised daily very early) will result in greater improvements in communication ability than Usual Care alone (UC), as measured by the WAB-R (AQ), ${ }^{2} 12$ weeks post stroke. 
6. UC-Plus (non-standardised daily very early) will result in greater improvements in communication ability than Usual Care alone (UC), as measured by the WAB-R (AQ), ${ }^{2} 26$ weeks post stroke.

7. VERSE (standardised, daily very early) aphasia therapy will result in greater improvements in connected speech than UC-Plus (non-standardised daily very early), as measured by Discourse Analysis, ${ }^{10-16} 12$ weeks post stroke.

8. VERSE (standardised, daily very early) aphasia therapy will result in greater improvements in connected speech than UC-Plus (non-standardised daily very early), as measured by Discourse Analysis, ${ }^{10-16} 26$ weeks post stroke.

9. VERSE (standardised, daily very early) aphasia therapy will result in greater improvements in connected speech than Usual Care alone (UC), as measured by Discourse Analysis, ${ }^{10-16}$ 12 weeks post stroke.

10. VERSE (standardised, daily very early) aphasia therapy will result in greater improvements in connected speech than Usual Care alone (UC), as measured by Discourse Analysis, ${ }^{10-16}$ 26 weeks post stroke.

11. UC-Plus (non-standardised daily very early), will result in greater improvements in connected speech than Usual Care alone (UC), as measured by Discourse Analysis, ${ }^{10-16} 12$ weeks post stroke.

12. UC-Plus (non-standardised daily very early), will result in greater improvements in connected speech than Usual Care alone (UC), as measured by Discourse Analysis, ${ }^{10-16} 26$ weeks post stroke.

\subsubsection{Analysis Methods:}

The outcome measure for all effectiveness analyses that involve WAB-R (AQ) ${ }^{2}$ will be \%MPR. ${ }^{16}$ For outcomes assessed at 12 weeks post stroke, this will be $\% \mathrm{MPR}^{16}$ at 12 weeks post stroke, as defined in section 3.5.1 above. For outcomes assessed at 26 weeks post stroke this will be \%MPR ${ }^{16}$ at 26 weeks as measured at baseline. That is,

$\% M P R$ at 26 weeks $=\frac{(A Q \text { at } 26 \text { weeks })-(A Q \text { at baseline })}{100-(A Q \text { at baseline })} \times 100$

The outcome measure for all efficacy analyses involving Discourse Analysis ${ }^{10,11}$ will be \%CIUs.

\subsubsection{Comparison between VERSE and UC-Plus groups:}

\subsection{Communication Outcomes (WAB-R $(A Q)^{2}$ :}

A longitudinal linear mixed model will be used to assess difference between the VERSE and UCPlus groups on $\% \mathrm{MPR}^{16}$ at 12 weeks and 26 weeks. The main between-group difference at each time point will be assessed through the interaction effect of group and timepoint. The model will adjust for baseline aphasia severity by including the WAB-R (AQ) ${ }^{2}$ score at baseline as a covariate. Baseline stroke severity will be controlled for by including the NIHSS ${ }^{4}$ at baseline as a fixed factor in the model. Recruitment site will be included as a random effect.

\subsection{Connected Speech Outcomes (Discourse Analysis):}

A similar longitudinal linear mixed model will be used to assess difference between the VERSE and UC-Plus groups on \%CIUs ${ }^{10-16}$ at 12 weeks and 26 weeks. The main between-group difference at each time point will be assessed through the interaction effect of group and timepoint. The model will adjust for baseline aphasia severity by including the WAB-R (AQ) ${ }^{2}$ score at baseline as a covariate. Baseline stroke severity will be controlled for by including the NIHSS ${ }^{4}$ at baseline as a fixed factor in the model. Recruitment site will be included as a random effect. 


\subsection{Communication Outcomes (WAB-R $\left(A Q^{2}\right)$ ):}

A longitudinal linear mixed model will be used to compare each of the VERSE and UC-Plus groups to the UC group on $\% \mathrm{MPR}^{19}$ at 12 weeks and 26 weeks. The main between-group difference will be assessed through the interaction effect of group and time point. The model will adjust for baseline aphasia severity by including the WAB-R (AQ) ${ }^{2}$ score at baseline as a covariate. Baseline stroke severity will be controlled for by including the NIHSS ${ }^{4}$ at baseline as a fixed factor in the model. Recruitment site will be included as a random effect.

\subsection{Connected Speech Outcomes (Discourse Analysis):}

A similar longitudinal linear mixed model will be used to compare each of the VERSE and UC- Plus groups to the UC group on \%CIUs ${ }^{10-16}$ at 12 weeks and 26 weeks. The main between-group difference at each time point will be assessed through the interaction effect of group and timepoint. The model will adjust for baseline aphasia severity by including the WAB-R (AQ) ${ }^{2}$ score at baseline as a covariate. Baseline stroke severity will be controlled for by including the NIHSS ${ }^{4}$ at baseline as a fixed factor in the model. Recruitment site will be included as a random effect.

\subsubsection{Handling of Missing Data:}

Missing data for secondary analyses will be handled as per section 3.5.3.

\subsection{Other Secondary Outcomes: Quality of Life}

It is hypothesised that:

Very early aphasia therapy (UC-Plus and VERSE) will result in better quality of life at 12 and 26 weeks post stroke than UC control.

\subsubsection{Outcome measure for Quality of Life}

Quality of life will be assessed using the Stroke and Aphasia Quality of Life Scale (SAQoL). ${ }^{17}$

\subsubsection{Statistical Hypotheses for Quality of Life}

The specified quality of life hypotheses involve the assessment of the following statistical hypotheses:

1. Very early daily aphasia therapy (UC-Plus or VERSE) will result in higher quality of life than Usual Care alone at 12 weeks post stroke.

2. VERSE aphasia therapy will result in higher quality of life than Usual Care alone at 12 weeks post stroke.

3. UC-Plus will result in higher quality of life than Usual Care alone at 12 weeks post stroke.

4. VERSE aphasia therapy will result in higher quality of life that UC - Plus at 12 weeks post stroke.

5. Very early intensive aphasia therapy (UC-Plus or VERSE) will result in greater improvements in communication ability at 26 weeks post stroke.

6. VERSE aphasia therapy will result in higher quality of life than Usual Care alone at 26 weeks post stroke.

7. UC- Plus will result in higher quality of life than Usual Care alone at 26 weeks post stroke.

8. VERSE aphasia therapy will result in higher quality of life that UC - Plus at 26 weeks post stroke. 


\subsubsection{Analysis Method:}

Separate linear mixed effects regression model will be used to assess quality of life as measured by the SAQoL ${ }^{17}$ at 12 weeks post stroke and at 26 weeks post stroke. Each model will compare the effects of:

1. VERSE therapy to usual care alone

2. UC- Plus therapy to usual care alone

The model will adjust for baseline aphasia severity by including the WAB-R (AQ) ${ }^{2}$ score at baseline as a covariate. Baseline stroke severity will be controlled for by including the NIHSS ${ }^{4}$ at baseline as a fixed factor in the model. Recruitment site will be included as a random effect. The treatment effects will be reported as difference in SAQoL ${ }^{17}$ (between VERSE and UC and between UC- Plus and UC); the corresponding 95\% confidence intervals will be reported.

\subsubsection{Handling of Missing Data:}

Handling of missing data will be similar to the detail in section 3.5.3.

\subsection{Other Secondary Outcomes: Cost Effectiveness}

It is hypothesised that:

Very early intensive aphasia therapy (UC-Plus and VERSE) will be more cost-effective than Usual Care at 26 weeks post stroke.

\subsubsection{Analysis Method:}

Details of the cost effectiveness analyses will be published separately and are not included here. Results from the effectiveness analyses will be the same for describing the effectiveness component of the incremental cost effectiveness calculation.

\subsection{Secondary Outcomes: Safety Important Medical Events (IME) Adverse Events (AE) and Serious Adverse Events (SAE)}

Important Medical Events (IMEs), Adverse Events (AEs) and Serious Adverse Events (SAEs) are defined in the main trial protocol. These are expected to be rare occurrences. Aphasia therapy is not expected to have an effect on these events. Therefore, no formal hypotheses have been stated about these outcomes. All IMEs, AEs and SAEs will be reported by therapy group.

As rare events, counts of IMEs, AEs and SAEs are expected to have a Poisson or negative binomial distribution. The distributions of these events will be modelled and appropriate generalised linear mixed models will be developed to assess differences between groups if the data suggests that there is a between-group difference of greater than 3 standard deviations in any of these event types.

\subsection{Sensitivity Analyses}

Sensitivity analysis for the primary outcome will be conducted under various assumptions about the missing data. The main analysis is planned under an assumption of missing at random; therefore, the sensitivity of the results to plausible departures from MAR will be explored as a part of an intentionto-treat analysis strategy. ${ }^{25-27}$

The 2010 National Research Council Panel on the Handling of Missing Data in Clinical Trials ${ }^{27}$ outlined two frameworks for modelling missing data: selection models and pattern-mixture models. The selection model framework models the probability of nonresponse (data being missing) rather than the distribution of the outcomes. In contrast, the pattern-mixture model framework models the distribution of the outcomes, under the assumption that the distribution of outcomes is different for responders and non-responders. The pattern-mixture model framework admits a transparent and easily-interpretable method for conducting a sensitivity analysis which involves adding a parameter 
(delta) to the mean response. The parameter, delta, measures the degree of departure from missing at random. In the VERSE trial, we propose using a pattern-mixture model to conduct a sensitivity analysis that assesses sensitivity of the results to plausible departures from the MAR assumption. If the inference about the treatment effects can be overturned by plausible values of the delta parameter, then the results of the trial will be considered equivocal.

We note that the gain in transparency and interpretability of conducting sensitivity analyses for pattern-mixture models may be offset by the computational complexity in averaging across large numbers of patterns in models with a large number of repeated measures. We do not anticipate facing this issue in the VERSE trial since outcomes are measured at only two timepoints after baseline (at primary endpoint and at follow up). However, if for any reason, we are unable to conduct a sensitivity analysis under the pattern-mixture model framework, the sensitivity analysis will be conducted under an appropriate selection model framework.

\subsection{Tables and Figures for the Main Paper}

The proposed tables and figures for the main results are presented in Appendix 1. Table 1 will report the main baseline demographic and stroke characteristics by participant group. Table 2 will report baseline communication, cognition and swallowing characteristics. Table 3 will report key summary data about the timing, frequency of therapy and amount of therapy provided in each group, length of hospital stay and discharge destination. Tables 4 and 5 will report the primary and main secondary outcomes at 12 and 26 weeks post stroke and Table 6 will report deaths and Serious Adverse Events. Figure 1 will be the CONSORT ${ }^{22}$ diagram. Figure 2 will be a bar chart of each grade on the mRS $^{5}$ in each treatment group at 12 and 26 weeks post stroke. Figure 3 will be a forest plot of the treatment effect on the primary outcome among different subgroups at 12 and 26 weeks post stroke.

\subsection{Approval}

The final version of this statistical analysis plan was approved by the Executive Committee on $20^{\text {th }}$ December 2017. 


\section{References}

1. Godecke E, Armstrong E, Ciccone N, Middleton S, Rai T, Holland A, Whitworth A, Rose M, Ellery F, Cadilhac D, Hankey GJ, Bernhardt J. The design of “A prospective multicentre, randomised controlled trial of Very Early Rehabilitation in Speech (VERSE) in patients with aphasia following acute stroke.” Intl J Stroke. 2016, 11(5):586-592.

2. Kertesz A. Western Aphasia Battery-Revised. New York: NCS Pearson; 2007.

3. Enderby PM, Wood VA, Wade DT. The Frenchay Aphasia Screening Test ( ${ }^{\text {rd }}$ Ed). London: Stass Publications; 2013.

4. National Institute of Health Stroke Scale (NIHSS). http://www.strokesite.org/stroke_scales/stroke_scales.html

5. Rankin J. Cerebral vascular accidents in patients over the age of 60. Scot Med J. 1957;2:200-215. [On-line]. Available: www.strokecenter.org

6. Bamford J, Sandercock P, Dennis M, Burn J, Warlow C. Classification and natural history of clinically identifiable subtypes of cerebral infarction. Lancet. 1991;337:1521-1526.

7. Ward EC, Conroy AL. Validity, reliability and responsivity of the Royal Brisbane Hospital Outcome Measure for Swallowing. Asia Pac J Speech, Lang Hear. 1999, Volume 4, Number 2: 109-129.

8. Perry A, Morris M, UnsworthC, Duckett S, Skeat M, Dodd K, Taylor N, Reilly K. Therapy Outcome Measures for Allied Health Practitioners in Australia: The AusTOMs. Int J Qual Health Car 2004 16,4:285-291.

9. Kaplan EF, Goodglass H, Weintraub S. Boston Naming Test ( $\left(2^{\text {nd }}\right.$ Ed.). New York: NCS Pearson. 2000.

10. Brookshire RH, Nicholas L. Presence completeness and accuracy of main concepts in the connected Speech of non-brain injured in adults. J Speech Hear Res, 1995;38:145-156.

11. Nicholas L, Brookshire RH. Presence completeness and accuracy of main concepts in the connected Speech of non-brain injured in adults. J Speech Hear Res, 1995;38:145-156

12. Boles L. Conversational discourse analysis as a method of evaluating progress in aphasia: A case report. J Com Dis. 1998;31:261-274.

13. Ellis C, Rosenbek JC, Rittman M R, Boylstein CA. Recovery of cohesion in narrative discourse after left-hemisphere stroke. J Rehab Res Dev. 2005; 42:6,737-46.

14. Godecke E, Ciccone N, Granger A, Rai T, West D, Cream A, Cartwright J, Hankey GJ. A comparison of aphasia therapy outcomes before and after Very Early Rehabilitation following stroke. Int J Lang Com Dis. March 2014, 49(2):149-61. DOI:10.1111/14606984.12074

15. Godecke E, Rai T, Ciccone N, Armstrong E, Granger A, Hankey GJ. Amount of therapy matters in very early aphasia rehabilitation after stroke: A clinical prognostic model. Sem Speech Lang. 2013,34,3:239-251.

16. Ciccone N, Cream A, West D, Cartwright J, Rai T, Granger A, Hankey GJ, Godecke E. A randomised controlled trial comparing individual and Constraint Induced Aphasia Therapy in very early recovery following stroke. Aphasiology, July 2015. DOI: 10.1080/02687038.2015.1071480

17. Hilari K. The Stroke and Aphasia Quality of Life scale 39 item version. Philadelphia: 2001.

18. Benaim C, Cailly B, Pélissier J, Pérennou D. Validation of the aphasic depression rating scale. Stroke, 2004;35:1692.

19. Lazar RM, Minzer B, Antoniello D, Festa JR, Krakauer JW, Marshall RS. Improvement in aphasia scores after stroke is well predicted by initial severity. Stroke, 41, 1485-1488.

20. R Core Team (2014). R: A language and environment for statistical computing. $R$ Foundation for Statistical Computing, Vienna, Austria. http://www.R-project.org/. 
21. IBM Corp. Released 2017. IBM SPSS Statistics, Version 25.0 Armonk, NY: IBM Corp.

22. Boutron I, Moher D, Altman DG, Schultz K, Ravaud P for the CONSORT group. Extending the CONSORT Statement to randomized trials of nonpharmacologic treatment: Explanation and elaboration. Ann Intern Med 2008;148:295-309.

23. Chan A-W, Tetzlaff JM, Altman DG, Laupacis A, Gøtzsche PC, Krleža-Jerić K, Hróbjartsson A, Mann H, Dickersin K, Berlin J, Doré C, Parulekar W, Summerskill W, Groves T, Schulz K, Sox H, Rockhold FW, Rennie D, Moher D. SPIRIT 2013 Statement: Defining standard protocol items for clinical trials. Ann Intern Med 2013;158:200-207.

24. Chan A-W, Tetzlaff JM, Gøtzsche PC, Altman DG, Mann H, Berlin J, Dickersin K, Hróbjartsson A, Schulz KF, Parulekar WR, Krleža-Jerić K, Laupacis A, Moher D. SPIRIT 2013 Explanation and Elaboration: Guidance for protocols of clinical trials. BMJ 2013;346:e7586.

25. White IR, Royston P, Wood AM. Multiple imputation using chained equations: issues and guidance for practice. Stat Med. 2011 Feb 20;30(4):377-99.

26. White IR, HN J, Carpenter J, Pocock SJ. Strategy for intention to treat analysis in randomised trials with missing outcome data. BMJ 2011; 342(d40).

27. National Research Council. 2010. The Prevention and Treatment of Missing Data in Clinical Trials. Washington, DC: The National Academies Press. https://doi.org/10.17226/12955. 
Appendix 1

Table 1: Baseline Participant and Stroke Characteristics

\begin{tabular}{ccccc}
\hline UC & UC+ & VERSE & All \\
& $n(\%)$ & $n(\%)$ & $n(\%)$ & $n(\%)$ \\
\hline
\end{tabular}

Recruitment Region

Australia

New Zealand

Patient Details

Age Median (IQR)

$<65$

65-80

$>80$

Gender

Male

Female

Pre-morbid History (Living arrangements prior to stroke)

Home alone

Home with other

Supported accommodation

Unknown

Stroke Risk Factors

HTN

IHD

AF

Hypercholesterolaemia

Diabetes

Smoking

Baseline NIHSS, Mean (SD)

Baseline mRS (binary)

Low (0-2)

High (3-6)

Oxfordshire Stroke Classification

TACS 
PACS

POCS

LACS

Haemorrhage

Time to Randomisation (days), Median (IQR)*

*n.b. time calculated from stroke onset to randomisation 
Table 2: Baseline communication, swallowing and cognition characteristics

\begin{tabular}{ccccc}
\hline UC & UC+ & VERSE & All \\
& $n(\%)$ & $n(\%)$ & $n(\%)$ & $n(\%)$ \\
\hline
\end{tabular}

Frenchay Aphasia Screening Test Median (IQR)

Baseline Western Aphasia Battery - Revised AQ Mean (SD)
Ceiling
(93.7 - 100)
Mild
(93.6 - 62.6)
Moderate $\quad(62.5-31.3)$
Severe
$(0-31.2)$

Baseline Discourse Measures

No. of Words, Mean (SD)

No. CIUs, Mean (SD)

\% CIUs, Mean (SD)

Boston Naming Test, Mean (SD)

\section{AuSTOMS}

Dysarthria

No impairment

Mild impairment

Moderate impairment

Moderate/severe impairment

Profound impairment

Apraxia of Speech (AoS)

No impairment

Mild impairment

Moderate impairment

Moderate/severe impairment

Profound impairment

Clock Drawing/Cog Test, Median (IQR)

RBHOMS 
Table 3: Hospital Stay and intervention Characteristics

\begin{tabular}{|c|c|c|c|c|c|}
\hline & & $\mathbf{U C}$ & $\mathbf{U C}+$ & VERSE & All \\
\hline \multicolumn{6}{|l|}{$\begin{array}{l}\text { Length of Inpatient Hospital Stay* } \\
\text { Median (IQR) }\end{array}$} \\
\hline \multicolumn{6}{|l|}{$\begin{array}{l}\text { Time to first therapy,* Median } \\
\text { (IQR) }\end{array}$} \\
\hline \multicolumn{6}{|l|}{ Received Therapy as Inpatient n (\%) } \\
\hline \multicolumn{6}{|l|}{ Met Intervention Compliance n (\%) } \\
\hline \multirow{2}{*}{$\begin{array}{l}\text { Intervention number of Therapy } \\
\text { Sessions, Median (IQR) }\end{array}$} & Usual Ward Care & & & & \\
\hline & Intense Therapy & & & & \\
\hline \multirow{2}{*}{$\begin{array}{l}\text { Frequency: Sessions per week, } \\
\text { Median (IQR) }\end{array}$} & Usual Ward Care & & & & \\
\hline & Intense Therapy & & & & \\
\hline \multirow[t]{2}{*}{ Length of Session, ${ }^{\#}$ Mean (SD) } & Usual Ward Care & & & & \\
\hline & Intense Therapy & & & & \\
\hline $\begin{array}{l}\text { Intervention period }{ }^{+} \text {Total Therapy } \\
\text { Amount } \wedge \text { Mean }(\mathrm{SD})\end{array}$ & $\begin{array}{l}\text { Usual Ward Care } \\
\text { Intense Therapy }\end{array}$ & & & & \\
\hline \multirow{2}{*}{$\begin{array}{l}\text { Week } 12 \text { Total Therapy Amount, ^ } \\
\text { Mean (SD) }\end{array}$} & Usual Ward Care & & & & \\
\hline & Intense Therapy & & & & \\
\hline \multirow{2}{*}{$\begin{array}{l}\text { Week } 26 \text { Total Therapy Amount, ^ } \\
\text { Mean (SD) }\end{array}$} & Usual Ward Care & & & & \\
\hline & Intense Therapy & & & & \\
\hline
\end{tabular}

n.b. * calculated in days ${ }^{+}$Intervention period defined as 35 days post randomisation; ${ }^{\#}$ calculated in minutes; $\wedge$ calculated in hours 


\section{Table 4:}

Outcomes at 12 weeks

UC UC+ VERSE ALL

\section{Primary Outcome Measure}

Western Aphasia Battery - Revised AQ, Mean (SD)

\% Maximal Potential Recovery (MPR)

\section{Secondary Outcomes}

Discourse Measures

No. of Words, Mean (SD)

No. CIUs, Mean (SD)

\% CIUs, Mean (SD)

Boston Naming Test, Mean (SD)

Stroke and Aphasia Quality of Life Scale, Mean (SD)

Aphasia Depression Rating Scale, Mean (SD) 
Table 5: Outcomes at 26 weeks

UC UC+ VERSE ALL

\section{Primary Outcome Measure}

Western Aphasia Battery - Revised AQ, Mean (SD)

$\%$ maximal potential recovery (\%MPR)

\section{Secondary Outcomes}

Discourse Measures

No. of Words, Mean (SD)

No. CIUs, Mean (SD)

\% CIUs, Mean (SD)

Boston Naming Test, Mean (SD)

Stroke and Aphasia Quality of Life Scale, Mean (SD)

Aphasia Depression Rating Scale, Mean (SD) 
Table 6: Adverse Events and Serious Adverse Events

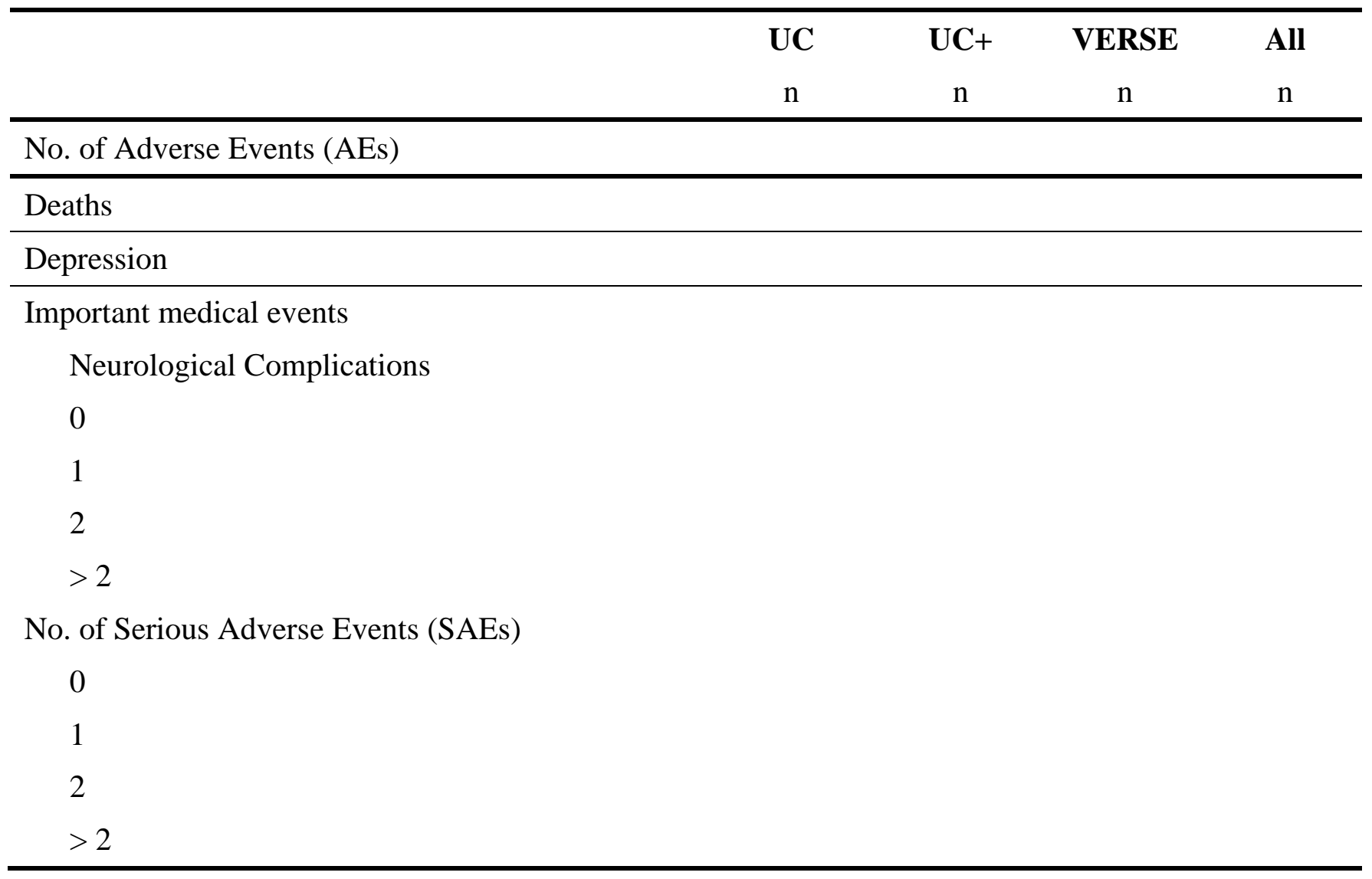


Figure 1. CONSORT Flow Diagram

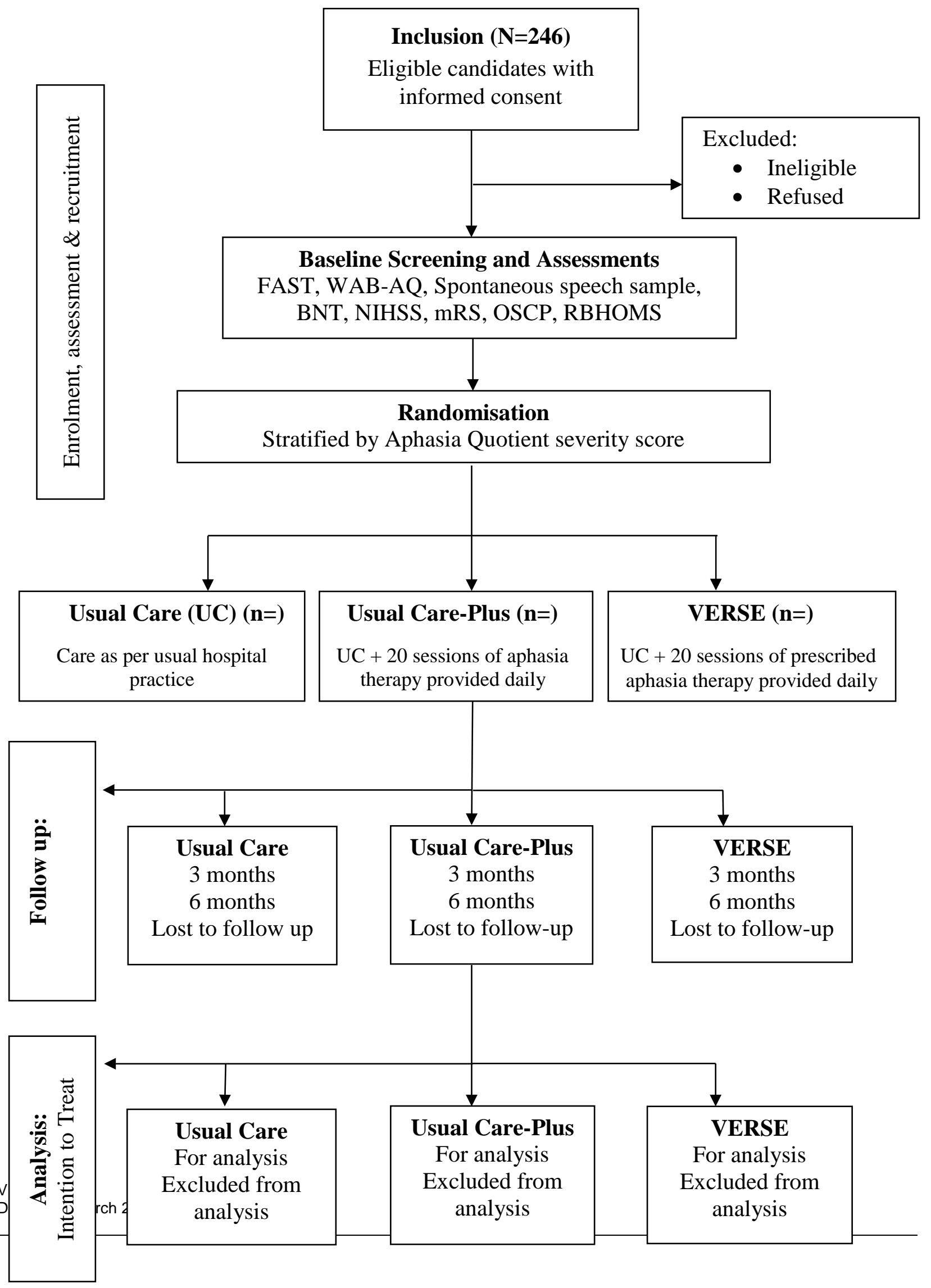


Figure 2. Figure 2. WAB-R (AQ) baseline, 12 and 26 week outcomes

Figure 3. Forest plot of treatment effect on primary outcome among different subgroups. 